\title{
Champlitte, haut-lieu de la vigne en Haute-Saône, à la croisée des territorialités
}

Champlitte, Mecca of vign in Haute-Saône standing at the parting of territorialities

Champlitte, Zentrum der Weinrebe in Haute-Saône, an der Wegscheide der Territorialitäten

\section{Sylvain Estager}

\section{OpenEdition}

Journals

Édition électronique

URL : http://journals.openedition.org/rge/3695

DOI : $10.4000 /$ rge.3695

ISSN : 2108-6478

Éditeur

Association des géographes de l'Est

Édition imprimée

Date de publication : 31 décembre 2012

ISSN : 0035-3213

\section{Référence électronique}

Sylvain Estager, «Champlitte, haut-lieu de la vigne en Haute-Saône, à la croisée des territorialités », Revue Géographique de l'Est [En ligne], vol. 52 / 3-4 | 2012, mis en ligne le 27 juin 2013, consulté le 08 septembre 2020. URL : http://journals.openedition.org/rge/3695; DOI : https://doi.org/10.4000/rge. 3695

Ce document a été généré automatiquement le 8 septembre 2020

Tous droits réservés 


\section{Champlitte, haut-lieu de la vigne en Haute-Saône, à la croisée des territorialités}

Champlitte, Mecca of vign in Haute-Saône standing at the parting of territorialities

Champlitte, Zentrum der Weinrebe in Haute-Saône, an der Wegscheide der Territorialitäten

Sylvain Estager

\section{Introduction:}

1 Des 14.000 hectares de vigne qui couvraient la Haute-Saône à la fin du XIXème siècle, il ne demeure plus aujourd'hui que des pratiques viticoles d'amateurs et une poignée d'exploitations commerciales en renaissance à partir des années 1970 (4 exploitations commerciales pour un total de 137 hectares). Champlitte, le premier vignoble à avoir tenté l'aventure de la replantation, est de loin le plus connu. Son histoire est avant tout liée à la célébration de la Saint-Vincent. Cette fête vigneronne, à fort contenu religieux, est effectivement à l'origine de l'entreprise de replantation et a su impulser une dynamique générant la principale activité de la commune.

2 Champlitte mobilise la vigne en tant que ressource territoriale et fait communément figure de haut-lieu de la vigne en Haute-Saône. Cette commune s'appuie sur une histoire viticole revisitée. Elle bouscule les identités et les mémoires liées à la vigne. Au final, Champlitte avec sa fête et son vignoble est en réalité à la croisée de territorialités complexes et parfois antagonistes. 
Doc 1 : Localisation de Champlitte en Haute-Saône

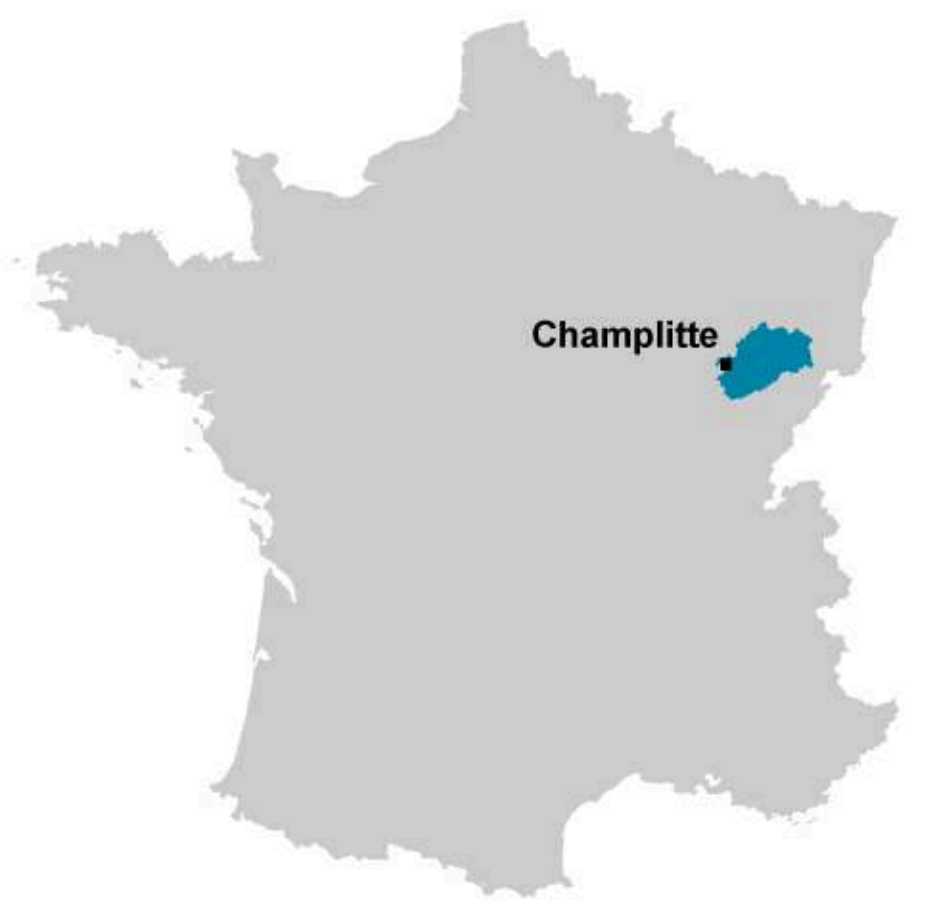

$100 \mathrm{~km}$

Carte : RGE, 2013

\section{Champlitte, la Saint-Vincent et les vignes}

\section{A. Une replantation originale}

3 L'origine de la replantation à Champlitte est assez singulière. L'initiative portée par quelques passionnés trouve à l'époque son inspiration dans une démarche mémorielle revendiquée. Le maintien d'une célébration religieuse, la Saint-Vincent, et l'activisme d'une personnalité locale sont à l'origine de la recréation de ce vignoble.

4 En 1941, A. Demard fonde un groupe folklorique "Les compares de Chanitte» pour redonner à la Saint-Vincent un lustre quelque peu perdu. Il est en effet receveur de la « Confrérie de Saint-Vincent » depuis 1936. En 1960, le groupe décide de replanter un clos en friche, à l'aide de cépages nobles, principalement du pinot noir. L'aventure de la replantation commence. La ressource de la tradition viticole de la commune est mobilisée et fédère rapidement autour d'un projet simple : refaire une production de qualité.

Champlitte a effectivement connu par le passé un vignoble de première importance. Jusqu'au début du XXème siècle, la vigne constitue une monoculture dans la commune et couvre jusqu'à 500 hectares $^{1}$ qui produisent des vins certes « un peu verts et un peu durs (...) mais droits et sains » (Guyot J., 1863), tout à fait « estimables » (Malte-Brun C., 1882).

6 Etabli en 1955/1956 pour le département ${ }^{2}$, le cadastre viticole recense encore à l'époque plus d'une centaine de déclarants amateurs : c'est sous cette forme de micro-parcelles, 
produisant à des fins d'autoconsommation familiale que la vigne s'est maintenue, mais le discours officiel qui accompagne la replantation mythifie l'entreprise comme s'il s'agissait d'un acte fondateur.

7 La véritable idée novatrice est de (re)tenter une aventure commerciale. Elle se concrétise par la création du Groupement Viticole Chanitois en 1974 qui appelle à souscription pour financer l'entreprise. Le succès est immédiat et grâce aux fonds récoltés auprès des 282 premiers souscripteurs ${ }^{3}$, dix hectares de vignes sont replantés sur les hauteurs de la commune. Deux ans plus tard dix nouveaux hectares agrandissent le vignoble. Celui-ci continue alors son expansion pour atteindre 34 hectares au milieu des années quatre-vingt dix.

8 L'exploitation est moderne et clairement à but commercial. L'objectif premier est celui de pérenniser l'entreprise. Le G.V.C fait appel aux méthodes du moment importées d'autres régions, de la Bourgogne voisine en particulier. Le vignoble a d'ailleurs été planté pour l'essentiel à l'écart du village sur des terrains qui n'avaient pour la plupart jamais été dédiés à cette culture.

9 La dimension historique, cet « héritage retrouvé » (Husson J-P., 2004), est intégrée pour justifier de l'existence d'une légitimité à cultiver la vigne dans la région, et donne au vignoble une profondeur dans le temps qui s'inscrit dans l'imagerie locale. Aujourd'hui les vignes produisent des vins de bonne facture (trois médailles d'or au salon de Paris depuis 2003) commercialisés sous l'appellation «Vin de pays de Franche-Comté-Coteaux de Champlitte ". Le succès est réel et un projet de classement en A.O.C. est envisagé, avec à terme, si cela se concrétise, la replantation de près de cent hectares supplémentaires.

10 En définitive, même si au regard des méthodes locales, ce vignoble n'a rien de traditionnel, « la tradition a été créatrice » (Demard J-C., 1986).

11 Signalons que sur ces terres, une démarche quelque peu différente a été entreprise par un viticulteur qui à l'origine appartenait au G.V.C. Avec l'envie de produire sous une forme plus traditionnelle et plus originale, $P$. Henriot a été l'un des premiers en France à tenter l'aventure d'une production «bio». Son exploitation couvre aujourd'hui 5 hectares et participe au rayonnement du vignoble en diversifiant la production.

12 Ainsi Champlitte est de loin la principale vitrine de la viticulture commerciale en Haute-Saône. Si deux autres exploitations ont depuis elles aussi tenté l'aventure ${ }^{4}$, elles ne bénéficient pas de la renommée de Champlitte qui de surcroît est portée par la célébration annuelle de la Saint-Vincent.

\section{B. La célébration de la Saint-Vincent}

13 Le maintien de cette célébration a participé à la renaissance du vignoble de Champlitte. Elle accompagne toujours l'entreprise en la rattachant au passé viticole de la commune. Ainsi elle procure une légitimité au vignoble, en lui donnant une profondeur historique.

\section{Organisation de la célébration}

14 Nous avons suivi quatre célébrations depuis 2005. L'organisation est rôdée et globalement reproduite chaque année de la même façon. 

l'événement rassemble le 22 janvier, selon les années et les rigueurs de l'hiver, entre 700 et 1000 personnes, essentiellement originaires du lieu. La ville est ornée de décorations multiples, mises en place par les autorités municipales et par plusieurs dizaines d'habitants qui prennent soin de parer leurs maisons de décorations multiples en rapport avec la célébration vigneronne. Les restaurants et cafés, affichent tous des menus festifs spécialement composés pour l'occasion. Ainsi, c'est l'ensemble de la commune qui célèbre la Saint-Vincent. La fête suit un cérémonial bien défini, ponctué de temps symboliques.

Vers neuf heures, les processions commencent dans les rues du village. Elles conduisent à un rendez-vous sur une petite place du village.

Doc 2 : Une célébration qui investit Champlitte

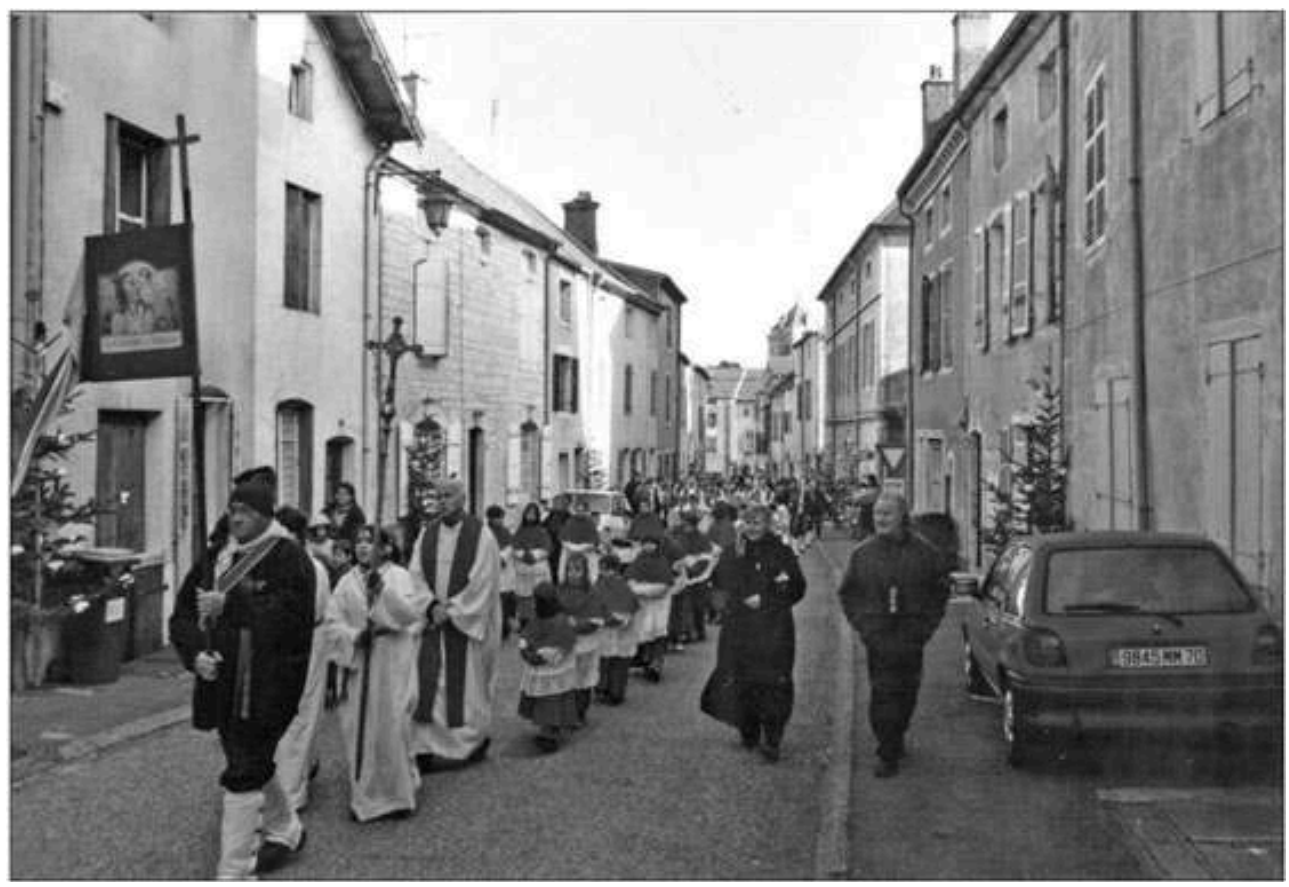

La procession emprunte les rues de la commune décorées aux couleurs de Champlitte pour

l'occasion. L'Eglise ouvre la marche, suivie par les membres de la confrérie, puis viennent les autres participants.

Photo : Estager, janvier 2006

17 Un premier apéritif est organisé depuis une dizaine d'années vers neuf heures et demi. Il rassemble près de six cents personnes autour d'un verre de Chardonnay local et victuailles préparées par les habitants du village. Ce bref instant de collation est marqué par la participation des élèves des écoles publiques de la commune habillés pour l'occasion en costume traditionnel de vigneron.

Aussi, la fête s'est quelque peu détournée d'un rite exclusivement chrétien. Ce moment se veut l'un des plus fédérateurs de la journée, malgré le froid matinal. D'ailleurs les instituteurs du village justifient la participation des écoles laïques à une fête religieuse par le fait que la tradition est ancrée dans la commune, qu'elle illustre de belle façon une leçon d'histoire locale et qu'il convient, selon eux, de ne pas faire preuve d'un 
rigorisme trop prononcé en matière d'idéal républicain. Ils soulignent par ailleurs qu'il y a encore quelques années les écoles fermaient toute la journée pour l'occasion.

Rapidement l'ordre du jour reprend sa place et vers dix heures, une nouvelle procession s'organise à travers la rue principale de la commune. Environ un tiers des personnes qui étaient présentes à l'apéritif s'en détournent pour rejoindre la place médiévale de Champlitte où se prépare la seconde collation. La marche lente et solennelle conduit à l'église où la messe va être célébrée.

L'église est bondée, près de cinq-cents personnes, et ne peut accueillir tout le monde. La messe est donc diffusée sur le parvis et la place de la mairie qui lui fait face, par plusieurs haut-parleurs installés pour l'occasion.

21 L'intérieur du bâtiment n'a pas été décoré pour la cérémonie du jour et seuls les membres de la confrérie en costume traditionnel témoignent du thème de la messe. Les prêches s'ouvrent à chaque fois sur la valeur chrétienne à accorder à l'office du jour. Le martyre de Vincent est rappelé. Le prêtre insiste sur la fidélité à la foi, sublimée par la résistance à l'abjuration et fait le pont avec l'actualité politique : canicule, malaise des banlieues, crise boursière et sociale sont interprétées, selon les années, comme le signe d'une société en mal de repères et de valeurs. C'est alors que la vigne est intégrée au propos. Elle est mise en scène comme une allégorie d'une société idéalisée : héritage transmis par les anciens, elle se nourrit de la tradition et du savoir-faire. Elle ne produit qu'à force de courage et d'abnégation, sous l'autorité et la bienveillance divine...

$\mathrm{Au}$ sortir de la messe, une nouvelle procession, moins solennelle que la précédente, conduit à travers les vieilles rues étroites, à l'ancienne place médiévale où le cortège retrouve le groupe qui n'a pas assisté à l'office religieux.

Une dizaine de stands distribuent alors vin blanc et vin rouge locaux. Plusieurs milliers de beignets, gaufres et autres brioches, réalisées par la population de la commune, sont distribués aux participants. Les organisateurs insistent sur la performance afin de mettre en évidence l'adhésion et l'importance de la mobilisation autour de l'événement. De brefs discours des autorités municipales, religieuses et de la confrérie, ponctuent une organisation désormais beaucoup plus festive que ce qui a été fait dans la matinée.

Le temps religieux a désormais cédé place à celui des festivités populaires et des références constantes au passé viticole. Les discours sont consacrés essentiellement au passé vigneron de la commune, qui jusqu'à présent n'avait été que très rarement évoqué.

On peut noter que ceux-ci sont prononcés depuis une estrade située devant la «maison espagnole ", remarquable et imposante bâtisse du XVIème siècle, érigée sur la place médiévale.

Ainsi, les références à la viticulture d'autrefois prennent place dans un cadre spécifique, assimilable à un écrin et qui peut s'interpréter comme la légitimation matérielle et spatialisée de cette dimension historique.

La fête se termine en éclatant les lieux où se produisent différentes actions : certains rejoignent l'église pour être intronisés au sein de la confrérie, d'autres demeurent sur la place médiévale et quelques uns commencent à rejoindre les salles des restaurants et les maisons où de conséquents repas franc-comtois seront servis le reste de la journée. 


\section{La Saint-Vincent dans les discours officiels et médiatiques}

28 Au niveau de la communication médiatique, l'événement est complètement pris en main. Les organisateurs prennent soin de mettre en scène une image consensuelle de la célébration. La confrérie, la municipalité, les publications locales et le traitement journalistique habillent les festivités d'un discours officiel qui entend avant tout donner une épaisseur historique à l'événement et un sens spirituel marqué. Ainsi la saint-Vincent est quelque peu déshabillée de sa dimension festive.

L'organisation de la fête la situe effectivement systématiquement dans une perspective historique ancienne. Dans chaque discours les références à l'histoire de la commune et surtout au passé viticole dominent le propos. L'épisode phylloxérique de la fin du XIXème siècle est présenté comme le tournant majeur dans l'histoire du bourg viticole qui endure alors les mêmes affres que le reste de l'hexagone. La célébration est dès lors présentée comme la capacité du lieu et de sa population à résister à la dynamique générale. Elle a résisté aux chocs multiples et conserve une inscription quasiidentitaire, forgée dans la communauté. Cette solidarité prétend trouver son illustration à travers la confrérie et la plantation récente du vignoble. Les vignes sont présentées comme l'expression finale de la dynamique engagée et le terme de « renaissance » n'est pas juste circonstanciel. Il se veut la conclusion d'un processus qui trouve ses origines au sein de l'histoire et des traditions locales.

30 "Les disciples de Saint-Vincent ont fait le serment de ne pas abandonner le vignoble (...), là où meurt le cep, renaît la vigne. Jamais cela n'a été aussi vrai qu'à Champlitte. Ici on marie le passé et le présent. » (J-C.Demard cité sur la place par l'un membre de la confrérie en 2006).

Enfin, si le propos peut sembler quelque peu amphigourique, il n'en est pas moins repris très largement par les médias locaux qui rendent compte de l'événement.

32 Une lecture du propos médiatique depuis 2005 à travers la presse et les informations télévisées régionales (Estager S., 2008) permet en effet de souligner que la version officielle donnée par les organisateurs à la célébration est bien relayée.

\section{Champlitte, haut-lieu de la vigne en Haute-Saône ?}

Champlitte est donc un lieu incontournable de la vigne en Haute-Saône. Le traitement médiatique de la Saint-Vincent conjugué à la renommée locale du vignoble et à la distribution de ses vins dans la plupart des grandes surfaces de la région, participent à assimiler cette modeste commune du département à son vignoble. Le vignoble chanittois est d'ailleurs aujourd'hui mis à l'honneur dans toutes les brochures touristiques qui traitent de la Haute-Saône.

Ainsi, à l'échelle de la Haute-Saône, Champlitte peut être assimilé plus spécifiquement à un haut-lieu.

Cette notion ne se limite pas à une dimension spirituelle omniprésente mais concerne tout lieu «érigé délibérément et collectivement au statut de symbole d'un système de valeur territoriale ». Il résulte d'une convection sociale qui associe le lieu à une image ou une idée et participe au construit identitaire et territorial (Debarbieux B., 1993). " Quintessence » du lieu (Gentelle P., 1995) ou «Paroxysme du lieu» (Piveteau J.L., 1993), le haut-lieu se caractérise par trois composantes: un espace clairement identifié, une valorisation de cet espace, chargé de symboles et le partage de la représentation symbolique de cet espace par un groupe plus ou moins large. Il permet de lire un 
territoire et de comprendre le système qui préside à sa constitution. En effet, la convergence des processus qui mettent en place la charge symbolique d'un haut-lieu ne tient pas du hasard; elle puise au sein des ressources disponibles du territoire et permet alors de les mettre à jour.

36 Au regard de cette définition, Champlitte apparaît pleinement comme un haut-lieu de la vigne en Haute-Saône :

37 - La commune, avec son vignoble, est un lieu qui présente de nombreuses manifestations du fait viticole: village à architecture vigneronne, nombreux clos, murgers et baraques de vigne vestiges de la viticulture passée, parcelles d'amateurs, toponymes en lien avec la vigne... Ce lieu est facilement identifié et circonscrit, sans toutefois avoir besoin d'être localisé pour être reconnu.

38 - La charge symbolique de la commune est évidente. Elle incarne la vigne par deux composantes incontournables : son vin de pays et les festivités de la Saint-Vincent. La puissance d'évocation de ces deux symboles de la vigne est d'autant plus prononcée qu'ils sont originaux dans la région. Le vignoble local est le premier à s'être reconstitué et associé à la saint-Vincent, il incarne largement le « vin de pays » et la renaissance du fait viticole dans la région.

39 - Enfin, cette charge symbolique est très largement relayée au sein de la Haute-Saône. La commune et le département font de Champlitte une vitrine de la viticulture passée et actuelle d'autant plus originale que la vigne est une culture marginale. Ici la marginalité se fait singularité et donne au lieu un caractère précieux. 
Doc 3 : Une vigne tenue « à l'ancienne » dans Champlitte

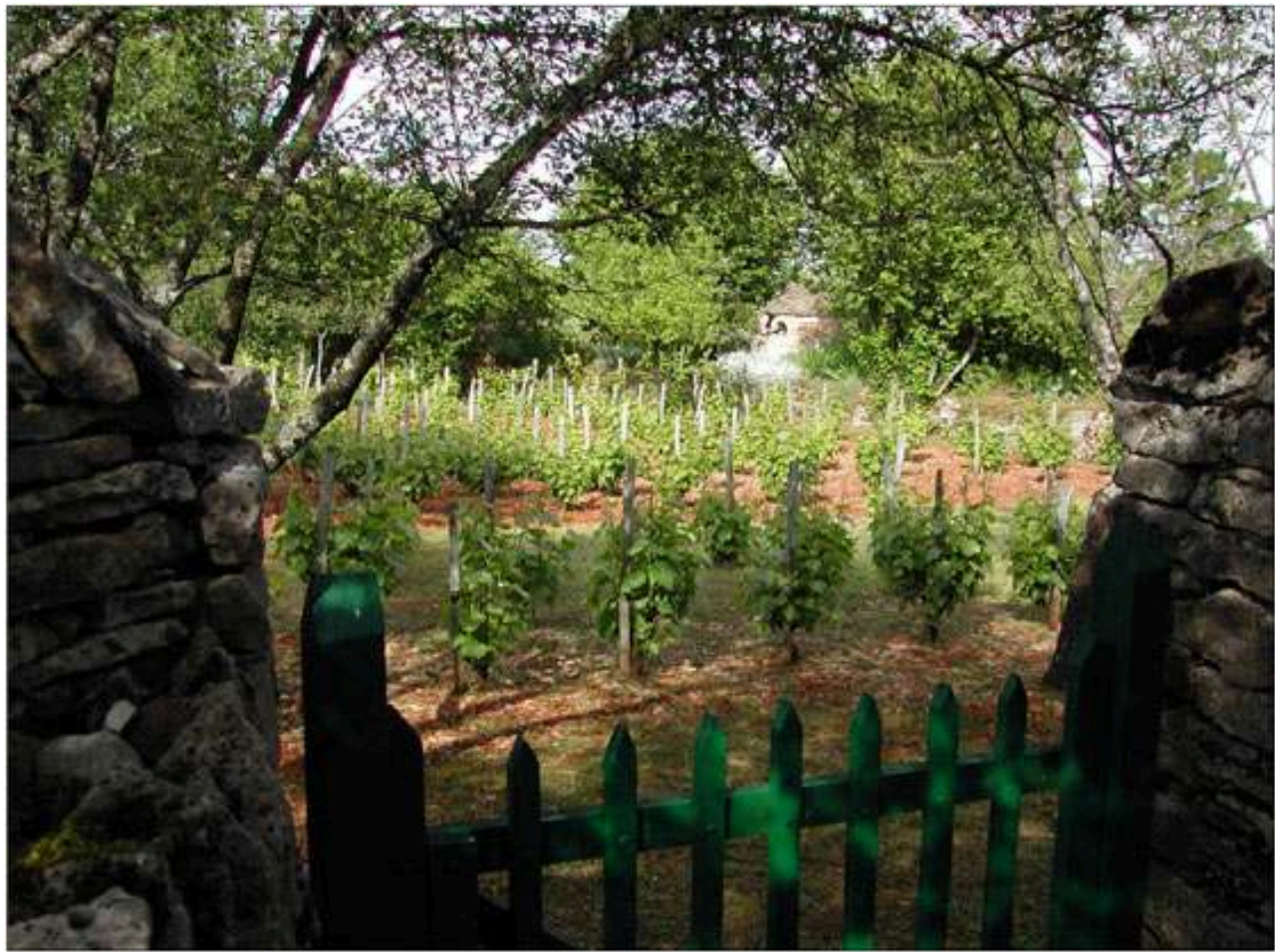

Une vigne remarquablement tenue selon des méthodes utilisées au début du siècle. Cette parcelle s'apparente à un sanctuaire de pratiques anciennes : cette vigne, située dans un clos se compose d'une centaine de ceps ; chacun est échalassé sur un bois fendu, en acacia et reçoit une taille basse la vigne est entièrement jardinée à la main et méticuleusement tenue ; remarquer la baraque de vigne à toit de pierre au fond de la parcelle.

Photo : Estager

Ainsi ce haut-lieu de la vigne en Haute-Saône est d'autant plus remarquable qu'il est unique. Il met en scène la vigne présentée comme une curiosité locale à fort contenu mémoriel : Champlitte est une fenêtre qui laisse entrevoir le passé viticole de la HauteSaône.En ce sens, la commune qui célèbre la vigne, est une mémoire du passé viticole de l'aire d'étude mais aussi une ouverture vers l'avenir à travers la réussite de son vignoble commercial. La vigne a été, est, et sera. A Champlitte, plus qu'ailleurs. La commune se pose alors comme le haut-lieu de la vigne en Haute-Saône.

\section{Un haut-lieu entre légitimité et artifices}

41 On ne peut aborder cette fête patronale comme un simple vestige de la tradition passée. Aujourd'hui la Saint-Vincent est avant tout une réalité de son temps, porteuse des valeurs de son époque.

\section{A. Une dimension traditionnelle en partie falsifiée}

Depuis deux décennies on assiste en France à une banalisation des fêtes, en partie liée à leur multiplication et à leur valeur marchande, politique ou publicitaire.

Les fêtes viticoles ne font pas exception et même dans des régions de vignoble reconnues, la récupération marchande et politique d'une histoire viticole locale 
falsifiée est souvent de mise (Aldhuy J., 2003). Ces fêtes qui s'inscrivent dans la tradition revendiquée, tendent à transformer l'événement en activité de loisir, proche du consommable (Di Méo G., 2005).

La véritable valeur de l'événement résiderait dès lors uniquement dans sa valeur actuelle, celle du quotidien ou de l'instant. La référence mémorielle ne serait qu'un alibi, une survivance revisitée qui rend compte non pas d'une tradition qui perdure mais d'un présent qui revisite le passé, le redéfinit, puis se le réapproprie. On rejoint ici la valeur que P. Nora donne aux «lieux de mémoire» (Nora P., 1983) ou qu'on peut associer à la " poussée mémorielle » accompagnant la redécouverte d'une culture rurale dans les années 1970 (Joutard P., 2003).

Les différentes composantes au sein du rite festif offrent une grille de lecture des valeurs territoriales proposées par l'événement : l'héritage traditionnel est là, en constante évolution, et il côtoie la construction actuelle de l'événement, toujours un peu artificielle et témoin de son époque (Bonnet-Carbonell J., Fournier L-S., 2004). Cela vaut en particulier pour les fêtes religieuses plus ou moins laïcisées. En proie aux syncrétismes de tous ordres et à une utilisation politique, au sens large du terme, elles sont souvent fort bien orchestrées.

La Saint-Vincent à Champlitte est effectivement à la croisée de ces différentes dynamiques. Il faut cependant distinguer, dans la mesure du possible, ce qui relève véritablement d'un héritage du passé de ce qui tient de la reconstruction historique. L'inscription historique de la fête ne doit pas nous abuser. Les organisateurs interrogés, ont d'ailleurs confirmé spontanément que les formes de la célébration avaient été " reprises en mains " depuis une quinzaine d'années. La fête a été en grande partie détournée de sa dimension festive, païenne voire paillarde. Il s'agissait là d'une volonté claire d'inscrire l'événement dans un cadre spirituel bien plus prononcé qu'il ne l'était autrefois.

47 Le propos justifie toujours la démarche en faisant référence à une "tradition » antérieure à celle reçue dans l'immédiat: c'est ainsi que la fête prétendument " traditionnelle " ne l'était plus véritablement aux dires de certains, car une "tradition " encore plus ancienne, par définition assez hypothétique, lui faisait défaut. La célébration actuelle a donc remplacé celle qui la précédait, au nom de la restauration de la dite «tradition», authentique celle-ci...

48 Les participants, issus du cru pour la plupart, ne sont pas dupes de la valeur à accorder à la Saint-Vincent. En particulier ceux qui l'ont connue au sortir de la guerre et qui l'ont vue évoluer. La mère d'un viticulteur septuagénaire que nous interrogions avec son fils résume bien un propos récurrent parmi les personnes interrogées:

49 «Cela n'a plus rien à voir avec les fêtes que j'ai connues, avec toutes leurs bondieuseries ... moi ça m'enlève même l'envie de boire un coup! »

50 Ainsi la Saint-Vincent aujourd'hui crée la tradition présente, assure peut-être en partie la future, mais remanie largement celle qui la précède.

51 Outre les différents discours, la démonstration s'appuie sur des symboles spatialisés forts et parfaitement définis : ruelles anciennes à architecture viticole, église, apéritif sur la place médiévale de la commune, le tout parcouru et habillé par les processions, les costumes anciens et une dose de folklore local. En bref, un ensemble visuellement marquant qui donne une tonalité différente aux espaces, aux bâtiments. On construit 
ainsi une mémoire, sur la base d'une histoire revisitée qui se structure à l'aide de symboles territoriaux et temporels puissants.

Le fait le plus remarquable, qui ne manque d'ailleurs pas de surprendre les visiteurs dans la commune, réside en l'absence visuelle du vignoble actuel aussi bien dans le déroulement de la Saint-Vincent que dans le paysage communal.

Les vignes d'aujourd'hui sont effectivement situées à l'écart de Champlitte et les seules que l'on aperçoit en se promenant dans le bourg, sont celles tenues par les viticulteurs amateurs: petits clos jardinés, treilles, ou micro-parcelles atypiques façonnées par un individu créatif... Cela tend à renforcer la démonstration de la continuité et de la légitimité à inscrire le vignoble actuel dans la lignée de celui d'autrefois en créant l'illusion qu'il demeure identique à ce qu'il était à l'époque: fi des immenses parcelles modernes, alignées et façonnées à la machine, à la couleur bleuâtre...

\section{B. Une fonction pacificatrice : la ville unifiée?}

Dans la plupart des cas, les fêtes communales, patronales en particulier, se singularisent par une sacralisation du territoire au sein duquel elles se déroulent : «cela affiche d'une certaine façon son inviolabilité (le territoire). Ils légitiment leur appropriation collective» (Di Méo G., 1999).

Le phénomène semble particulièrement clair à Champlitte. La fête établit une sorte de raccourci spatial et temporel qui tend à transformer le cadre communal en un sanctuaire dédié à la vigne et à son âge d'or passé. L'appropriation des lieux est manifeste. En dépit des oppositions, la Saint-Vincent est incontestablement dotée d'une puissance fédératrice puisque y participent même ceux qui la critiquent. Certes, au moment de la messe par exemple, le groupe se scinde. Mais au final, le cortège religieux rejoindra sur la place médiévale ceux qui n'ont pas voulu participer aux prêches et autres manifestations spirituelles. Il ne s'agit pas là d'une pacification célébrée de façon démonstrative et cathartique comme lors d'un carnaval, mais d'une unification autour de la Saint-Vincent et de la vigne.

Enfin certains cadres de notre identité nationale collective, qui passent dans de nombreuses manifestations par le respect des codes républicains, sont balayés lors de la célébration. La place centrale bordée par la mairie et l'église diffuse la messe. La représentation municipale est assurée lors de la célébration et la Mairie s'associe pleinement à l'événement. De même, les écoles publiques y participent. De fait, en cette vieille terre de tradition radicale, la séparation des Eglises et de l'Etat n'est plus de mise, le jour de la Saint-Vincent. Le territoire est lors de cette fête avant tout celui de la commune et il s'organise selon des cadres et des codes qui ne répondent qu'à la seule logique locale. La parenthèse créée pour l'événement est spatiale, temporelle et politique.

57 L'un des aspects territoriaux de cette fête réside donc dans la profonde redéfinition de la société qui se réinvente un cadre, une organisation et une identité en dehors des références juridiques et sociétales habituelles. La dimension festive permet de faire apparaître une dynamique syncrétique à même de véhiculer les thèmes associés à la Saint-Vincent, pour au final, singulariser la commune et son rapport à sa propre histoire. En apparence, elle gomme pour un temps les clivages sociaux, culturels ou politiques. Le message idéologique trouve dans la valeur religieuse œcuménique affirmée, un argument supplémentaire pour créer le mythe et fait basculer les valeurs 
prônées par la fête dans l'ordre des représentations qui désormais s'impose à celui des réalités du quotidien.

L'illusion qui en ressort permet d'ailleurs de mettre en perspective l'actualité nationale ou internationale. Les prêches n'hésitent pas à parler de cohésion sociétale, de mixité sociale et entendent par moment donner la leçon, arguant de la réussite de l'événement à l'exemple de ce propos prononcé lors de l'édition 2006, en prélude à l'apéritif offert sur la place médiévale:

"En ces heures tristes où nos enfants s'égarent ${ }^{5}$, nous montrons ici que le respect des valeurs, de notre histoire et l'attention à autrui permettent de souder une communauté ".

Ces artifices rhétoriques, nous le verrons, sont parfois rejetés avec rudesse et l'on peut en outre remarquer qu'en dépit du discours officiel qui célèbre la fraternité, l'organisation de la célébration légitime certaines élites politiques, sociales ou religieuses en les situant toujours en bonne place dans les cortèges ou lors des discours. L'ordre social est en fait juste un peu bousculé par les coutumes et la grammaire spatiale qui structurent l'événement.

61 Au final, la commune semble tout de même unie, au moins le temps de l'événement, par et pour la Saint-Vincent. Si le mécanisme fonctionne globalement, c'est sans doute parce qu'il répond à une attente de la population, parfois en proie à un manque de repères identitaires

\section{Un refuge identitaire dans le cadre d'une époque post- moderniste?}

62 Nombre de fêtes patronales ou agraires sont l'expression d'une recherche identitaire au sein d'une société en perte de repères. Ces fêtes portent souvent la marque d'une volonté de trouver un repère clair dans le cadre d'une époque post-moderniste en proie à une profonde déstructuration identitaire pour certaines populations, en particulier au sein d'un milieu rural vieillissant comme ici en Haute-Saône (Bonnet-Carbonell J., Fournier L-S., 2004). Ce constat rejoint celui de P. Nora lorsqu'il présente ce qu'il appelle la fin de l'histoire-mémoire, comme une carence affective qui entraîne l'apparition de multiples lieux mémoriels, le plus souvent en basculant d'une échelle nationale ou régionale, à une autre beaucoup plus fine, d'ordre local ou communal (Nora P., 1983).

63 La Saint-Vincent telle qu'elle est célébrée revêt une profonde âme identitaire. Plus qu'un phénomène de résilience classique, la fête perdure et bénéficie d'un succès porté par l'envie de créer un ancrage symbolique. La Saint-Vincent se structure d'ailleurs localement en se concentrant sur la population du village. La fête ne s'ouvre aux communes environnantes que par l'intermédiaire des confréries vigneronnes. L'identité affirmée ici est donc claire : la fête est chanittoise et/ou vigneronne. Il s'agit d'un lieu de mémoire enraciné dans le local. La force de ce genre de représentation repose sur son caractère unificateur (Becker A., 2001) : unité de temps (ici le 22 janvier), unité de lieu (ici Champlitte) et unité d'action (ici la célébration). La Saint-Vincent, avec ses nombreuses références à l'âge d'or viticole, participe à la création et à la nostalgie d'une société de l'époque embellie mais fondamentalement désincarnée, sortie de son temps, et surtout débarrassée de ses maux, de sa rudesse et de ses malheurs. La crise viticole de la fin du XIXème siècle apparaît comme une fatalité qui a brutalement 
détruit les vignes florissantes de l'époque et la Première Guerre mondiale, l'expression de valeurs de courage et de mérite... La réalité des faits est largement mythifiée. Il est possible que ce passé réinventé traduise la peur de l'oubli et l'incapacité à traduire le présent, le quotidien en terme de valeurs, d'identité et surtout de perspectives. La Saint-Vincent constitue dès lors un refuge identitaire réactionnaire face à son époque, dans un département qui se marginalise au sein du territoire national. Elle procède ainsi de l'artifice dénoncé par P. Nora lorsqu'il aborde ces lieux de mémoire.

\section{Un haut-lieu, qui ouvre sur des territorialités multiples}

\section{A. La saint-Vincent ne fait pas l'unanimité}

Les valeurs qui accompagnent la mise en scène de ce haut-lieu de la vigne en HauteSaône sont facilement identifiables. Mais leur généralisation ne préjuge en rien de leur acceptation dans l'aire d'étude et même parmi le cortège des participants.

Les enquêtes par entretiens et par questionnaires réalisées ${ }^{6}$ ont permis de dégager les grandes lignes de la perception de Champlitte en qualité de haut-lieu de la vigne en Haute-Saône. Le vignoble est un «lieu-attribut» (Debarbieux B., 1995) pour Champlitte, quasiment systématiquement associée à la vigne.

Deux critiques qui se rejoignent ont été exprimées par près de la moitié des chanittois interrogés ${ }^{7}$ qui participent à la fête. Elle est jugée trop repliée sur elle-même. Aucune publicité n'est faite pour l'événement et la volonté de rester «entre-nous » pour ne pas "perdre l'esprit de la célébration» est clairement revendiquée par les organisateurs rencontrés. Cette critique se conjugue à une lecture plus politique qui stigmatise une coloration religieuse et réactionnaire de la célébration. On reproche pêle-mêle, l'abandon de la dimension païenne qui existait autrefois et la reprise en main par les autorités religieuses. Enfin, signalons qu'à plusieurs reprises la célébration fut taxée de pétainisme avec comme argument principal la prise en main de la confrérie par l'initiateur de la replantation du vignoble, en 1941.

67 Aussi l'adhésion à la Saint-Vincent ne saurait se mesurer à la seule aune de la participation et de la mobilisation car il existe un véritable sentiment critique quant à la dimension politico-religieuse. Notre approche ici, se limite à exposer des tendances mises à jour sur les lieux.

\section{B. Un haut-lieu rejeté par les viticulteurs non chanittois}

Les enquêtes par entretien effectuées auprès des viticulteurs amateurs dans l'ensemble $\mathrm{du}$ département soulignent une perception de la Saint-Vincent et du vignoble de Champlitte assez remarquable. Précisons tout d'abord que, pour la plupart des personnes interrogées, il est très difficile de dissocier la fête, du vignoble de la commune. Une question sur la Saint-Vincent amène de façon systématique une réponse qui dévie sur le vignoble et inversement. Ceci tend à démontrer que l'habillage officiel qui accompagne l'entreprise du viticole est bien relayé au sein de la région. L'amalgame fonctionne. 
à accepter Champlitte comme haut-lieu de la vigne. La teneur politico-religieuse des
prêches qui accompagnent la célébration suscite en effet une réprobation importante
en dehors de la commune. Visiblement marqués par leur formation au sein des
premiers cycles de l'Ecole de la République, ces viticulteurs amateurs assimilent la
forme actuelle de la Saint-Vincent à une logique de résistance face à la laïcisation de la
société. On rejoint ici les critiques formulées par certains participants à la fête. Il existe
sans doute une forme de jalousie, relevant de l'esprit de clocher, qui participe à la
formulation des ces critiques. Néanmoins il est clair que le rejet de Champlitte comme
haut-lieu de la vigne n'est pas simplement instinctif. Il procède souvent d'une position
réfléchie et solide.

propos devient effectivement beaucoup plus clair et argumenté lorsque la question de la qualité du vignoble est abordée. La viticulture, telle qu'on la pratique à Champlitte, n'est absolument pas représentative de la tradition viticole locale. C'est sans doute ce qui vaut à Champlitte la condamnation la plus unanime (par les trois quarts des personnes interrogées). La vigne cultivée en Haute-Saône est, de longue date, le fruit d'un travail essentiellement d'amateur, en général dans le cadre d'une tradition familiale. Jardinée, soignée, cette viticulture est singulière et chargée d'affectif. De fait, la viticulture commerciale mise en œuvre à champlitte contraste par 
la mécanisation généralisée et les techniques utilisées. Il est dès lors logique d'y voir à l'origine, une pratique qui ne correspond pas aux savoir-faire locaux.

Presque tous les propos recueillis témoignent que le sujet est particulièrement sensible pour les viticulteurs de la région. Dès lors on conteste l'idée de renaissance du vignoble portée par la célébration de la fête patronale. Une dichotomie assez nette apparaît entre les anciens viticulteurs de la région et l'entreprise viticole chanittoise.

L'appréciation sur le vin produit est clairement induite par ce regard sévère et rares sont ceux qui ont fait preuve d'un minimum d'indulgence sur sa qualité. Le jugement est souvent très rude. Citons le cas d'un viticulteur d'une soixantaine d'années que nous interrogions en présence de sa mère. Alors qu'il nous expliquait que parce qu'il était réalisé de façon «industrielle ", le vin de Champlitte ne pouvait être de bonne qualité, il se vit interrompre brutalement par son aïeule:

« Non, il n'est pas mauvais, il est carrément imbuvable !...Tiens, il est encore pire que le nôtre! ».

79 Ainsi, le sentiment qu'inspirent la Saint-Vincent et le vignoble de Champlitte est parfois véritablement cinglant, sans retenue, ni nuance. Cette réalité est assurément liée à la dimension affective que revêt la culture de la vigne chez beaucoup. La fête patronale est un lieu de mémoire, vivant par définition, qui bouscule, ravive ou exacerbe certains sentiments en rapport avec la culture de la vigne dans l'aire d'étude. Elle se comporte comme un révélateur du rapport que certains entretiennent avec la mémoire et la pratique viticole.

\section{Champlitte, un haut-lieu de la vigne à la croisée des territorialités}

Champlitte, son vignoble et sa célébration vigneronne s'inscrivent donc différemment au sein des territoires de l'aire d'étude et participent différemment, selon les échelles d'approche à la définition des territoires de la zone d'étude et en premier lieu, à celui de Champlitte.

81 - A l'échelle de la commune, la vigne est une composante majeure du territoire. Champlitte se confond largement avec son vignoble et la Saint-Vincent.

82 - A l'échelle de la Haute-Saône, Champlitte est un haut-lieu de la vigne. Vitrine viticole du département, la commune s'habille d'un discours officiel qui en fait un symbole de l'histoire, de la mémoire et de la richesse du territoire.

83 - A l'échelle de la communauté des viticulteurs amateurs de l'aire d'étude, Champlitte ne peut prétendre fédérer sur son image et incarner un lieu emblématique du fait viticole. Le rejet est manifeste et la mise en scène du symbole incarné n'est pas acceptée.

84 La fête permet la production de symboles territoriaux. Le choix de ces symboles qui relève de la phase d'organisation de la fête impose à l'arrivée une image qui marque non seulement la fête elle-même, mais qui conditionne aussi la définition de l'ensemble de la commune, au delà du seul jour de la célébration.

85 Il est clair qu'à Champlitte l'identité affirmée s'appuie presque exclusivement sur des symboles territoriaux en lien avec la société agraire et qu'au delà de la comptabilité statistique, la commune se revendique avant tout rurale.

86 Les images de la commune que véhicule la fête, par l'intermédiaire de photographies anciennes ou actuelles (mais la plupart en noir et blanc), de croquis ou d'aquarelles 
réalisés par un artiste local, célèbrent la commune comme un espace rural à spécialisation viticole qui pourrait correspondre à une réalité du XVIIIème ou du XIXème siècle. Le décalage entre cette idéologie et une certaine réalité vécue, notamment par les gens du cru, explique sans doute le ressentiment prononcé à l'égard de la Saint-Vincent. D'ailleurs, le département, dans ses brochures touristiques ou par le relais de la presse régionale présente la Saint-Vincent et le vignoble chanittois comme un héritage de la tradition viticole de la région mais insiste surtout sur la dimension folklorique de la fête, ce qui tend à aller à l'encontre du propos et de l'identité revendiquée par les organisateurs de l'événement. L'événement est détourné sitôt que l'on sort du cadre communal.

On perçoit donc le décalage qui se manifeste immédiatement entre la mythification qui accompagne l'événement et la perception qui peut en être faite en dehors de Champlitte.

En dehors de la commune où elle est fêtée, la Saint-Vincent bouscule les mémoires, en particulier celles liées à la vigne, plus qu'elle ne les accompagne.

\section{Conclusion}

Le principe même d'une célébration telle que la Saint-Vincent est de distiller en une journée, une concentration de production émotionnelle et affective en lien avec un passé idéalisé. L'événement réduit le temps et ancre dans les mémoires, non seulement les festivités du 22 janvier mais aussi un concentré d'histoire en rapport avec la viticulture de jadis.

90 La brièveté de l'événement et son aspect festif permettent de faire fi des raccourcis historiques et apaisent les oppositions qui pourraient s'exprimer sur ce point. L'image de Champlitte et de la Saint-Vincent est alors relayée, utilisée et reprise au sein de l'aire d'étude pour participer à l'image de la Haute-Saône. Ainsi, la mémoire de chaque viticulteur, chanittois ou non est censée se retrouver dans ce discours officiel : le local et l'intime doivent se diluer dans ce raccourci général qui traduit la volonté d'affirmer une identité locale dans un cadre globalisé qui s'impose à tous.

91 Ne nous trompons pas sur la valeur à accorder à ce haut-lieu de la vigne dans la région. Il relève largement de l'artifice et ne peut prétendre incarner un symbole porté par la tradition viticole locale. Il faut donc voir à travers Champlitte une réalité du présent et non un legs porteur d'une tradition conservée. C'est un lieu de mémoire comme les a définis à l'origine $\mathrm{P}$. Nora. Sa réalité et les dérives qui l'accompagnent donnent à Champlitte tout son sens pour comprendre son rôle dans la construction territoriale.

92 L'aspect parfois artificiel de ces hauts-lieux ou de certains événements qui s'y apparentent est courant (Debarbieux B., 2000). Il convient de chercher derrière la symbolique spatialisée mise en scène dans ces hauts-lieux, la multiplication des lieux ou de pratiques plus banales mais essentielles pour comprendre le territoire. Appréhendés séparément ces lieux n'ont que peu de puissance symbolique mais rassemblés derrière une entité à forte charge d'évocation, ils permettent de lui donner son statut de haut-lieu. Peu importe si le processus qui le met en place procède de l'artifice ; si le haut-lieu existe c'est qu'il répond à une attente. Il marie des territorialités publiques ou privées, qui demandent à être mises à jour. 

derrière Champlitte il faut comprendre que la vigne existe ailleurs. Qu'elle appartient au territoire de l'aire d'étude, à son histoire et aux mémoires. Si ce haut-lieu est mis en scène il procède en réalité de la convergence de nombreux lieux disséminés au sein du territoire.

L'atomisation de ces lieux, leur marginalité spatiale et leur appartenance majoritaire à la sphère privée ne permet pas de les doter d'une puissance symbolique qui assure leur reconnaissance dans l'aire d'étude. Aussi Champlitte remplit une fonction de représentation du fait viticole et trouve dans sa qualité de haut-lieu l'expression de cette fonction. Derrière ce haut-lieu il faut donc chercher d'autres lieux. Sa seule présence est une invitation faite au géographe à explorer et à comprendre ce qui existe au-delà.

\section{BIBLIOGRAPHIE}

Aldhuy Julien, 2003, « Production culturelle et émergence de lieux. Du vin et du jazz dans le haut Entre-Deux-Mers ", in Lieux de culture, culture des lieux, Gravari-Barbas M., Violer P. (dir.), Rennes, P.U.R., 306 p, pp. 157-165.

Becker Annette., « La Grande guerre, entre mémoire et oubli » in Cahiers français, La mémoire entre histoire et politique, Leonard Y. (dir.), $n^{\circ} 303$, Paris, La Documentation française, juilletaoût 2001, 224 p., pp.85-86

Bonnet Carbonell Jocelyne, Fournier Laurent-Sébastien.,2004, Fêtes et rites agraires en Europe : Métamorphose ?, Paris, L'Harmattan, 269p.

Crozat Dominique,2005, « De la fête aux loisirs : événement, marchandisation et invention des lieux »,Paris, Annales de géographie n6, p.307-328.

Debarbieux Bernard, 1993, « Du haut-lieu et du Mont-Blanc en particulier », Paris, L'Espace géographique, $\mathrm{n}^{\circ} 1$, p.5-13.

Debarbieux Bernard, 1995, «Le lieu, le territoire et trois figures de rhétorique », Paris, L'Espace géographique, $\mathrm{n}^{\circ} 2$, p. 97-112

Debarbieux Bernard, 2000, « Qu'avait-il donc d'incroyable ce pique-nique? », Paris, L'Espace Géographique n4, p.363.

Demard Albert, 1962, "La fête du ban et le maintien de vignes à Champlitte ", Haute-Saône magazine $\mathrm{n}^{\circ} 16, \mathrm{p} 10-14$.

Demard Jean-Claude. et Paignard Marcel, 1986, La Saint-Vincent à Champlitte, Besançon, Ed. La Lanterne, $80 \mathrm{p}$.

Di Méo Guy, 2001, « Le sens géographique des fêtes », Paris, Annales de géographie, n622, pp 624-644

Di Méo Guy, 2005, « Le renouvellement des fêtes et des festivals, ses implications géographiques » , Paris, Annales de Géographie, n643, p. 227-243. 
Estager Sylvain, 2008, Un objet entre marginalité et territoire: la vigne en Haute-Saône, Thèse de Doctorat, Université de Lille 1, 481 p.

Estager Sylvain, «Comprendre une marginalité paysagère et son sens territorial. L'exemple de la vigne en Haute-Saône », Blois, Actes des deuxièmes Journées d'Etude du Paysage, E.N.S.N.P. décembre 2009. Gentelle Pierre, 1995, « Haut-lieu », L'Espace géographique n², p. 135-138.

Guyot Jules, 1864, Sur la viticulture du Nord-est de la France, Paris, Imp. Impériales, 287p.

Husson Jean-Pierre, 2004, « La vigne dans l'agglomération de Nancy, de l'objet relique au projet de renaturation ", Nancy, Revue Géographique de l'Est, 1-2

Joutard Philippe, 2003, « L'histoire en sabots », L'Histoire n²76, pp. 64-68

Malte-Brun Conrad, 1882, La France illustrée, tome 3, 693p..

Nora Pierre, 1983, Les lieux de mémoire, Paris, Gallimard, tome 1, pp13-24.

Piveteau Jean-Luc, 1993, « A propos des hauts-lieux », Paris, L’Espace géographique n¹, p 5-13.

\section{NOTES}

1. Source : A.D.H.S., Phylloxera 86 M2àM8

2. Source : A.D.H.S., delta 110.14

3. Les souscripteurs sont à prés de $90 \%$ chanittois. On peut estimer qu'environ un foyer sur trois a participé à la création du G.V.C. dans cette commune qui compte à l'époque environ 2000 habitants.

4. A Charcennes et à Roche \& Raucourt

5. Il s'agit d'une référence implicite aux événements de «l'automne chaud » de 2005 qui venait de s'achever dans certaines banlieues françaises.

6. Quarante-trois viticulteurs amateurs ont été interrogés lors d'enquête par entretien consacrés à la pratique viticole d'amateur dans la région (Estager S., 2008) . Le cas de Champlitte, a toujours été abordé.

7. Nous avons interrogé entre 10 et 15 personnes, de façon aléatoire, à chaques célébrations auxquelles nous avons assisté $(2005,2006,2008$ et 2010).

\section{RÉSUMÉS}

Champlitte, commune rurale de Haute-Saône est aujourd'hui connue pour ses vignes et la fête patronale de Saint-Vincent qui lui est associée. Ce vignoble est dans le département le premier à avoir tenté l'aventure de la replantation dans les années 1970. L'histoire de la replantation comme l'organisation de la fête patronale sont singulières et mobilisent toute la commune et ses habitants. La vigne est ici convoquée en tant que ressource historique du territoire local. Elle constituait, jusqu'au début du XXème siècle une quasi-monoculture dans la commune, avant de disparaître en tant que culture commerciale. Cette culture a depuis repris sa place au cœur de la vie communale et forge l'identité même de Champlitte. Celle-ci peut même être considérée comme un haut-lieu de la vigne à l'échelle de la Haute-Saône. La vigne est 
finalement prétexte à l'exposé de valeurs territoriales affirmées. Le thème viticole et la célébration de la Saint-Vincent offrent dès lors une clé de lecture sur ces valeurs qui par ailleurs sont diversement appréciées. Champlitte et sa célébration de la vigne témoignent au final d'un processus de production d'idéologies territoriales et des enjeux qui en découlent.

In Haute-Saône the rural town Champlitte is today known about its vigns linked to Saint-Vincent religious feast. In the seventies this vineyard is the first in the department to innovate by replanting vigns. The story of replantation as the organisation of the religious feast are singular and all the town and its inhabitants are concerned by. Vign is here considered as an historical resources from territory. Until the beginning of the XXe century it was almost a monoculture in Champlitte then this commercial culture diseaspears to become again today fundamental giving the town its identity. Champlitte can even be considered as the Mecca of vign for Haute-Saône. In fact, vign is a pretext staging his own local wine story enables to expose territorial values. Wine theme and Saint-Vincent celebration lead to understand those values wich are apprecied or

rejected. Finally, Champlitte and the Saint-Vincent celebration is an exemple of a process producting territorials ideologies and its stakes.

Champlitte, Landgemeinde in Haute-Saône, ist heutzutage bekannt für ihre Weinrebe und für das berufsständische Saint-Vincent Fest, das dazu gehört. Diese Weinrebe ist im Bezirk die erste, die das Abenteuer der Wiederverplanzung in den Jahren 1970 probiert hat. Die Geschichte der Wiederverplanzung ist wie die Organisierung des berufsständischen Festes eigenartig, und beide mobilisieren die Gemeinde und deren Bewohner. Die Weinrebe ist hier als geschichtliche Quelle des örtlichen Territoriums hervorgerufen. Sie bestand bis zum Anfang des zwanzigsten Jahrhunderts aus einer quasi Monokultur in der Gemeinde, bevor sie als Handelskultur verschwand. Diese Kultur hat seither ihre Stelle im Herzen des Lebens der Gemeinde wiedergewonnen und prägt die Identität von Champlitte. Diese Gemeinde kann sogar als Zentrum der Weinrebe in ganz Haute-Saône betrachtet werden. Die Weinrebe ist also Vorwand, um behauptete Territorialwerte auszustellen. Das Weinthema und das Saint-Vincent Fest geben nunmehr eine Schlüssel der Erklärung über diese Werte, die übrigens verschieden geschätzt werden. Champlitte und das Fest der Weinrebe beweisen schliesslich, wie allmählich Territorialideologien und die davon abgeleiteten Einsätze hergestellt werden.

INDEX

Schlüsselwörter : Champlitte, Haute-Saône, Saint-Vincent, territorium, Weinrebe, Zentrum

Keywords : Champlitte, Haute-Saône, mecca, Saint-Vincent, territory, vineyard

Mots-clés : Champlitte, haut-lieu, Haute-Saône, Saint-Vincent, territoire, vignoble

\section{AUTEUR}

\section{SYLVAIN ESTAGER}

Professeur agrégé - Docteur en Géographie - Université de Lille 1 - s.estager@free.fr 